\title{
Bemerkungen zu der Notiz E. Bataillon's: »Sur l'évolution de la fonction respiratoire chez les œufs d'Amphibiens:.
}

\author{
Von
}

\author{
E. Godlewski jun.
}

Eingegangen am 27. Mai 1901.

Durch die Giite des Herausgebers dieses Archivs wurde mir ein $\Lambda$ bzug der vorstehenden Mittheilung vorgelegt.

Es thut mir sehr leid, dass bei der Durchsicht der bisherigen Litteratur die interessante Arbeit von Prof. E. Bataillow ron mir iibersehen wurde, und dass aus diesem Grunde die Resultate derselben von mir nicht citirt und beriicksichtigt wurden. Ich benutze daher die Gelegenheit, um auf die von BaTAILLON 1) angegebene Methode der quantitativen Bestimmung der ausgeschiedenen Kohlensäure (l. c. pag. 731 und 306 der zweiten Arbeit) hinzuweisen. Die Methode ist wegen ihrer Einfachheit sehr leicht durchfihrbar und kann fiir entwickelungsphysiologische Untersuchungen, besonders bei Eiern, die gegen Gehalt von Baryt im Wasser wenig empfindlich sind, bei der Bestimmung der Quantität $\mathrm{CO}_{2}$ von großer Bedeutung sein.

Gleichzeitig will ich zugeben, dass E. BATAILlon der Erste war, welcher quantitative Untersuchungen über die ausgeathmete $\mathrm{CO}_{2}$ bei den Amphibieneiern angestellt hat. Es freut mich sehr, dass die unabhingig von einander nnd nach verschiedenen Methoden ansgefïhrten Versuche Ziffern ergaben, die so genau mit einander ibereinstimmen; ich muss jedoch bemerken, dass die von Bataillon aus meiner Arbeit (dieses Archiv. Bd. XI. pag. 585) entnommenen Ziffern sich auf die Kohlensäureproduktion verschiedener Eierportionen beziehen. Sie weisen also auf die Schwankungen (les oscillations) hin, welche bei den Eiern von verschiedenen Individuen, nicht aber von verschiedenen Entwickelungstadien einer und derselben Eierportion sich feststellen lassen.

Was die Sauerstoffabsorption anbelangt, so glaube ich nicht, dass man zum Schlusse berechtigt wäre: sque l'absorption d'oxygène montre des oscillations de même ordres. Wir finden nämlich in den Arbeiten von BA'taILLon

1) Bataillon, E., Evolution de la fonction respiratoire chez les embryons d'Amphibiens et de Téléostéens (C. R. et Mém. de la Soc. de Biologie. 1896. T. 48. pag. 730). - Nouvelles recherches sur les mécanismes de l'évolution Arclı. de Zool. Expérim. S. III. T. Y. pag. 281. 1897). 
keine quantitativen Angaben darüber. Die von ihm vorgeschlagene Methode der quantitativen Bestimmung des Sauerstoffs, welche sich für anderes Material als sehr vortheilhaft erweist, ist meiner Ansicht nach für die Untersuchung des embryonalen Gaswechsels bei Amphibieneiern nicht geniigend genau und hat zahlreiche Nachtheile. Diese Methode ist eine Modifikation des in der Pflanzenphysiologie öfters angewandten Verfahrens und ich habe Gelegenheit gehabt, bei meinen Versuchen 9-11 die Wirkung dieser Methode kennen zu lernen. Die Nachtheile dieses Verfahrens habe ich in meiner Arbeit (l. c. pag. 607) näher besprochen und will desswegen hier darauf nicht mehr eingehen. Aus demselben Grunde (vgl. pag. 607 meiner Arbeit) glaube ich nicht, dass die von Batalllon ans meiner Arbeit entnommenen Ziffern in Bezug auf Sauerstoffabsorption seinen Ansichten Stiitze verleihen können.

Ich habe jedoch fiir Sauerstoffabsorptionsbestimmung eine andere, meiner Ansicht nach, genauere Methode (pag. 609) benutzt. In diesen Bestimmungen, welche mit dem Apparat von Bossier und Makgis unternommen wurden, und die in dieser Hinsicht allein maßgebend sein können, finden wir in dem Entwickelungsgang einzelner Eierportionen in der Qnantitiit des absorbirten 0 keine Schwankungen. Die Vermnthung, dass savec des opérations trop espacées* sich die Schwankungen gegenseitig ausgleichen, muss erst mit Ziffern bewiesen werden.

Ich will bei dieser Gelegenheit anf einen Druckfehler hinweisen, der in der Bezeichnung der Abbildungen meiner Arbeit vorgekommen ist: auf 'Taf. XXI soll Fig. 10 als 18 , anf' 'Taf. XXII Fig. 18 als 10 bezeichnet werden. 